\title{
Numerical analysis method of chemical anchorage based on the
}

\section{cohesive zone model}

\author{
aYongquan Wang, ${ }^{b}$ jian Jing, ${ }^{\mathrm{C}}$ Tianjiao Chen
}

${ }^{a}$ College of Water Conservancy and Hydropower Engineering, Hohai University, Nanjing 210098, China

${ }^{b}$ College of civil and transportation engineering, Hohai University, Nanjing 210098, China

${ }^{\mathrm{C}}$ College of Water Conservancy and Hydropower Engineering, Hohai University, Nanjing 210098,

China

KEYWORD: Chemical anchorage; Pullout analysis; Numerical simulation; Cohesive zone model; Parametric analysis

ABSTRACT: In order to precisely analyze the mechanical performance of chemical anchorage, a new method using cohesive zone model to simulate the bond layer between reinforcement and concrete interface has been put forward. The three-dimensional model of ABAQUS constructed was based on chemical anchorage. In this model, the cohesive zone model with a certain thickness was used for bonding interface, the constitutive relation of which fell on the bilinear stress-opening displacement model. The influence of parameters on the load-displacement curves of single steel drawing was analyzed. The conclusion indicated that the ultimate bearing capacity of the structure (the maximum load of the load-end) was mainly determined by the fracture strength. The bearing capacity increases with higher fracture strength, and the fracture toughness has little influence on the ultimate bearing capacity of anchorage.

\section{INTRODUCTION}

Chemical anchorage has the most extensive application in anchorage connection technology, the stress distribution feature and anchorage performance of which are the hotspot and difficulty in the research. In the earlier studies, Cook ${ }^{[1]}$ and his team adopted a single reinforcement drawing method, where a large amount of experiment and a lot of labor and cost are required. Since the continuous development of the computer technology, numerical simulation method, based on computer technology, has become a new method to research the performance of chemical anchorage force ${ }^{[2-5]}$. In the simulation of chemical anchorage system, axisymmetric plane model ${ }^{[2,}$ 5], and three-dimensional entity model ${ }^{[3,4]}$ are widely adopted by scholars. In terms of the simulation method of glue layer, Cook and his team considered the corresponding layer thickness unit and the relative layer in establishing axisymmetric model. However, it is difficult to accurately obtain the constitutive of glue layer. Thus, in the following study, the researchers, like Bajer Miroslav, simulated bonding face directly by setting up unit of different spring stiffness. The spring stiffness is generally determined according to the measured load displacement curve. 


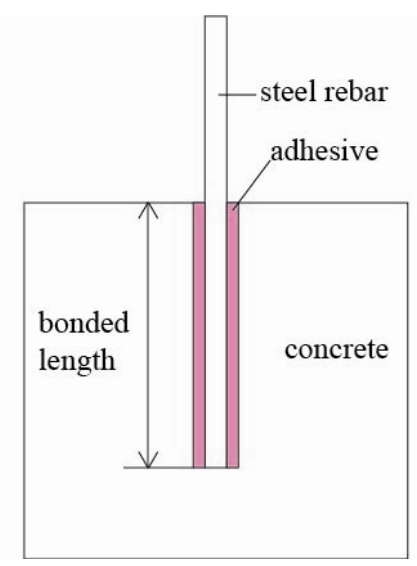

Figure 1. Chemical anchorage system

It was found that adding springs was complicated in the spring simulation method in ABAQUS modeling and the nonlinear springs must be added in INP files, which greatly increases the modeling workload, especially for 3D model. The cohesive force unit used to simulate the bond interface has good new development in recent years ${ }^{[6-9]}$. The cohesive zone model is mostly used in the analysis of the interfacial bond performance between FRP and concrete. For instance, Feng-chen $\mathrm{An}^{[6]}$ analyzed the three fracture modes between FRP and concrete, respectively opening mode, sliding mode and tearing mode. It comes to a conclusion that the simulation method is capable of properly describing the damage evolution and the process of the full cross section.

Based on the application of the cohesive zone model in the study on bond performance of FRP concrete interface, the numerical analysis of single reinforcement pullout analysis made an introduction of 3D bilinear cohesive zone model. And the related parameters of cohesive zone model were determined by test data.

\section{COHESIVE ZONE MODEL}

Cohesive zone model is a calculation method which is frequently applied and studied in fracture theory. From the view of energy, the method bypasses the complexity of the end of the crack and acquires the interfacial fracture energy directly through the external forces acting on the system. Currently, the widely used law of the relationship between tension and displacement mainly consists of bilinear, trapezoid, polynomial and exponential relationship ${ }^{[7-8]}$. Regardless of which relationship to be chosen, there must be two parameters that are taken into consideration: the traction-separation law must consider two cohesion parameters, one of which is the peak stress values in the traction-separation law curve called the ultimate shear stress (fracture strength), the other is the area surrounded by traction-separation law curve and the horizontal axis called energy release rate (fracture toughness or fracture energy) ${ }^{[9]}$.

The bilinear cohesive zone model is so simple in form and can be easily realized by finite element method that it is widely applied in the numerical simulation. The bilinear cohesive zone model is adopted in this paper to simulate the adhesive interface stress performance of chemical anchorage. Bilinear cohesive zone model has five control parameters: the interface fracture toughness $G_{f}$, the relative displacement in the interface when the interface begins to yield $\delta_{1}$, the relative displacement in the interface when completely destroyed $\delta_{f}$, the initial interfacial shear stiffness $K_{0}$, and the ultimate shear stress (fracture strength) $\tau_{f}$. The bilinear cohesive zone model and its parameters relationship are shown in Figure 2.

The interface fracture toughness can be expressed by the energy of the unit area as shown in formula (1), which can be determined by the experiment.

$G_{f}=\frac{1}{2} \tau_{f} \delta_{f}$ 
Where $\tau_{f}=$ ultimate shear stress (fracture strength); $\delta_{f}=$ the relative displacement of the interface when completely destroyed.

The initial interfacial shear stiffness:

$$
K_{0}=\frac{\tau_{f}}{\delta_{0}}
$$

Where $\delta_{0}=$ the relative displacement corresponding to the maximum shear stress.

The cohesive element unit (cohesive elements) is adopted to realize bilinear cohesive constitutive model. The material properties of the adhesive layer can be obtained by inputting the interface toughness, the tensile strength and the initial interfacial shear stiffness.

In the bilinear constitutive model, the damage level of adhesive materials can be described by the variable $D$ and it can be determined by the following formula ${ }^{[7]}$ :

$D=\frac{\delta_{f}\left(\delta_{m}-\delta_{0}\right)}{\delta_{m}\left(\delta_{f}-\delta_{0}\right)}$

Where $\delta_{m}=$ the maximum relative displacement achieved in the loading process.

There is no damage in the adhesive layer when $D$ is equal to zero, while there is damage failure when $D$ is equal to one.

$D=0$ layer material without damage, $D=1$ layer material damage failure. In the linear softening stage where the adhesive layer material reaches the cracking strength, the damage variable $D$ increases from 0 to 1 .

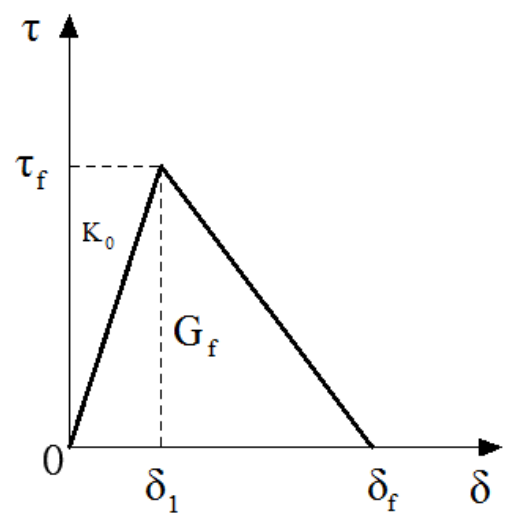

Figure 2. bilinear cohesive force model

\section{ESTABLISHMENT OF THREE -DIMENSIONAL COHESIVE ZONE MODEL}

\section{Material properties}

\section{Concrete and steel bar}

In the model, concrete and steel bar are both considered as the elastic material and parameters including elastic modulus and Poisson's ratio, which need to be input. In this paper, the elastic modulus of concrete is equal to $29.5 \mathrm{GPa}$ and the Poisson's ratio is equal to 0.2 ; the elastic modulus of reinforced materials is equal to $210 \mathrm{GPa}$ and the Poisson's ratio is equal to 0.3 .

\section{Bonding interface material}

There are two kinds of material models when the cohesive element is applied to simulate the bonding interface of the chemical anchorage. One is based on the continuum description and the other is based on the description of Traction-Separation. The latter is adopted in this paper. In this method, the linear elastic stage before the material reaches the ultimate strength and the stiffness softening stage after the material reaches the ultimate strength are given. The maxs damage criterion in ABAQUS was adopted in the paper. The criterion of crack propagation indicating the 
total failure of the cohesive element refers that the fracture energy of the element reaches the critical value or the displacement value reaches the ultimate failure value.

\section{Element types}

(1) The element types of concrete and reinforcement

Solid element C3D8R is used to simulate the concrete and reinforcement in the three-dimensional cohesive zone model. In this case, the calculation result of displacement is more accurate and the shear locking is uneasy to generate. When the large deformations occur to the gird, the analysis accuracy is seldom affected.

(2) The element types of the interface layer

The three-dimension element $\mathrm{COH} 3 \mathrm{D} 8$ which has eight nodes is used to simulate the cohesive element. In such case, the cohesive element is considered to be a two dimensional element and two facets separated by a certain thickness. These facets are connected with other solid elements respectively which only consider the out-of-plane force, including the normal stress and the shear stress in the direction of $\mathrm{XZ}$ and $\mathrm{YZ}$. The interface layer element with a certain thickness is adopted in order to simulate anchorage interface layer more realistically so that the debonding failure of the layer can be shown more intuitively.

\section{The establishment of the overall model}

Based on the composite model of ABAQUS, the establishment of interface layers mainly includes the following two methods: 1) Firstly, a complete structure is to be established. Then a thin layer is to be cut off to simulate the cohesive element. The cohesive force element share the public nodes with the other element to transfer the force and displacement. 2) The solid models of cohesion layer and other structural components are to be established. The unit displacement and stress of both sides of the cohesive element are coordinated through the binding constraints. The first method is adopted in this paper. Three dimensional finite element calculation model and element mesh are as shown in Figure 3.

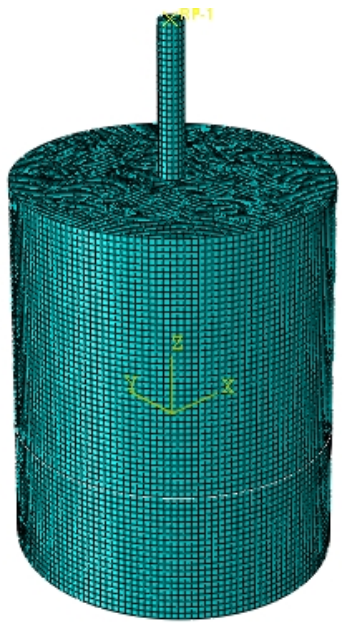

Figure 3. three dimensional finite element model

\section{INFLUENCE OF INTERFACE PARAMETERS ON LOAD -DISPLACEMENT CURVE}

For single pull-out test of chemically planted bar, the ultimate shear stress (fracture strength) $\tau_{f}$ and energy release rate (fracture toughness) $G_{f}$ are main cohesive parameters. So this thesis explores both of these cases: 1) keep the fracture toughness value of 0.8 , alter the fracture strength; 2) keep the breaking strength value of $30 \mathrm{MPa}$, change the fracture toughness. Load-displacement curve based on the different parameters is as shown in Figure 4.1. 


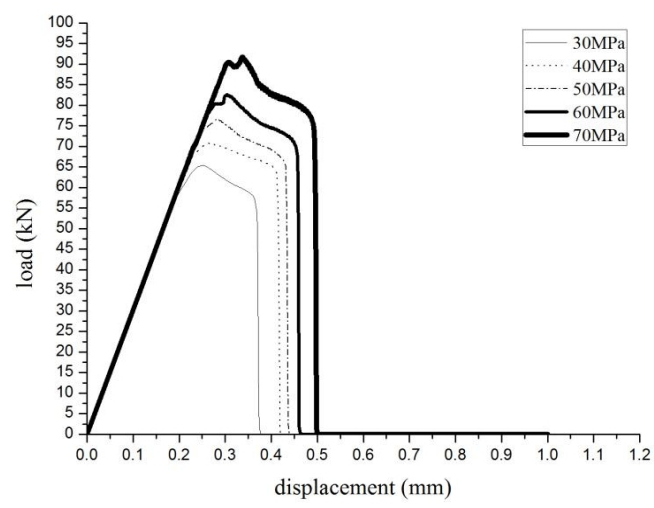

(a) Keep the fracture toughness value, alter the fracture strength

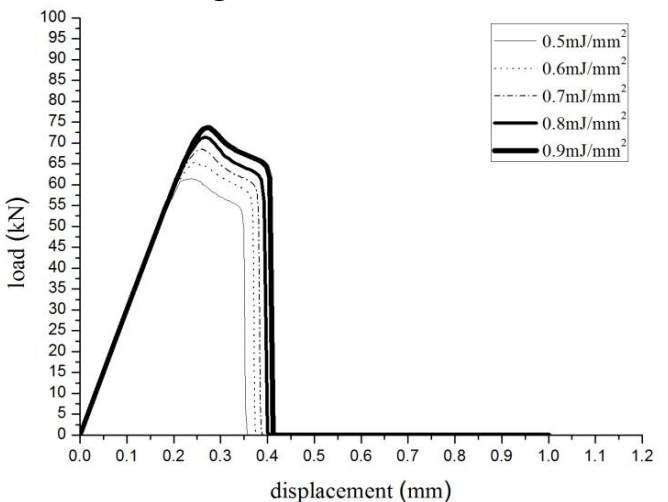

(b) Keep the breaking strength value, change the fracture toughness

Figure 4. Load-displacement curve of the loading end of the 3D cohesive model

Figure 4 illustrates that the interface will experience different stages in single pull-out test of chemically planting steel bars: elastic stage $(E)$, elastic + yield stage $(E+P)$, yield + de-bonding stage $(\mathrm{P}+\mathrm{D})$ and de-bonding stage $(\mathrm{D})$.

When the adhesive interface in the elastic stage, the slope of the load-displacement diagram in the linearly rising stage will not be influenced by changes of fracture strength and fracture toughness, which is determined by the cohesive element shear modulus; with the increase of load, when the adhesive interface in elastic + yield stage, fracture strength change has great influence on slope of load-displacement curve, while fracture toughness has a slight effect on it. The fracture strength is the main control factors for the ultimate load-carrying capacity of the structure. As the fracture strength increases, ultimate bearing capacity increases, the growth presents an increasing tendency; as the fracture strength is constant, the ultimate bearing capacity is improved along with the increase in the small value range of fracture toughness, but continual increase leads to the smaller change of ultimate bearing capacity, ultimate bearing capacity keeps nearly unchanged, when the fracture toughness reaches a certain value.

Fracture strength is also one of the most critical influences on the maximum displacement of the interface complete rupture. Remained fracture toughness unchanged, the maximum displacement of anchorage section complete de -bonding interface is increasing with the increase of the fracture strength, and increase greatly. Keep breaking strength, change the fracture toughness, with the increase of the fracture toughness value, the interface displacement of bar section complete de-bonding changes smaller and smaller, when the fracture toughness reaches a certain value, the maximum displacement of the interface hold steady.

Above all, fracture strength decide the ultimate bearing capacity of anchorage structure, and influence the load-displacement curve slope in elastic +yield stage, besides, the maximum displacement of the interface complete de-bonding is also severely affected; fracture toughness is little effect on the load-displacement curve, with fracture toughness increases, anchorage load displacement curve is closer and closer. 


\section{CONCLUSIONS}

This paper presents a new chemically planted bar numerical simulation method, which uses bilinear cohesive zone model to simulate steel bar and concrete bonding interface; the above parameter, analyses are proved to obtain the effects of fracture toughness and fracture strength cohesion parameters on load displacement curves. The following are main results that have been put forward.

(1) In a cohesive zone model. First of all, to establish a complete structure, cutting out a thin layer to simulate the cohesive element, cohesive force unit and other units of public nodes, and in order to transfer the force and displacement, the convergence of the method of modeling is easy and convenient.

(2) Fracture strength is the main factor that determines the ultimate bearing capacity (end of the loading maximum load), and with the increase of the fracture strength, anchorage limit bearing capacity increases. The ultimate bearing capacity of anchorage is less affected by the fracture energy. Slope of linear rise section of the load displacement curve in elastic stage is depended on cohesive element shear modulus.

\section{ACKNOWLEDGEMENTS}

The authors are grateful for the National Natural Science Foundation of China for Youth ( 51409084 ) and Natural Science Foundation of Jiangsu province for Youth (BK20130836).

\section{REFERENCES}

Cook R A. Behavior of Chemically Bonded Anchors [J]. Journal of Structural Engineering, ASCE, 1993(9):2744-2762.

McVay M, Cook R A, Krishnamurthy K. Pullout Simulation of Post-Installed Chemically Bonded Anchors [J]. Journal of Structural Engineering, ASCE, 1996(9): 1016-1024.

Eligehausen R, Cook R A, Appl J. Behavior and Design of Adhesive Bonded Anchors [J]. ACI Structural Journal, 2006, 103(6): 822-831.

Miroslav Bajer, Jan Barnat. The glue-concrete interface of bonded anchors [J]. Construction and Building Materials, 2012(34):267-274.

Priyank Upadhyaya, S.Kumar. Pull-out capacity of adhesive anchors: An analytical solution[J]. International Journal of Adhesion \& Adhesives, 2015(60):54-62.

Feng-chen An. Modelling of FRP-concrete interfacial bond behavior[D], university of edinburgh,2015.

CHANDRA N , LI H , SHET C, et al . Some issues in the application of cohesive zone models for metal-ceramic interfaces[J] . International Journal of Solids and Structures , $2002,39(10)$ : 2827-2855 .

PARK K, PAULINO G H, ROESLER J R . A unified potential-based cohesive model of mixed-mode fracture $[\mathrm{J}]$. Journal of the Mechanics and Physics of Solids , 2009 , 57(6) : 891-908 .

ANYFANTIS K N , TSOUALIS N G . A novel traction-separation law for the prediction of the mixed mode response of ductile adhesive joints[J]. International Journal of Solids and Structures , 2012, 49( 1) : 213-226.

CAMANHO P P, DAVILA C G . Mixed-mode decohesion finite elements for the simulation of delamination in composite materials [R] . Washington D.C.: NASA , 2002 . 
